\title{
Periodic and Aperiodic Close Packing: A Spontaneous Hard-Sphere Model
}

\author{
B. W. van de Waal \\ Twente University of Technology, Chemical Physics Laboratory, P.O. Box 217, 7500 AE Enschede, The Netherlands
}

The study of small aggregates of atoms or molecules constitutes a new, exciting, and rapidly growing field. In recent years techniques have been developed to produce, isolate, and examine very small particles, consisting of from only a few to several hundreds of atoms (1). Such particles (commonly referred to as clusters or van der Waals molecules) are of fundamental interest, with properties intermediate between those of isolated (gas-phase) atoms and those of the bulk solid. Their structure is believed to deviate considerably from that of the crystalline state, with important consequences for thermodynamic and electronic properties.

Apparently, the periodicity, characteristic of the bulk crystal structure, may be only poorly developed, or even completely absent in small clusters, to give way to other tendencies, more important in this size domain but inconsistent with three-dimensional periodicity, like five-fold symmetry.

\section{Construction of a Model}

Structural features of clusters (and of small crystalline fragments) can be illustrated using ordinary party balloons and a set of Ping-Pong ${ }^{\mathrm{TM}}$ balls. The balloons should be of good quality, so as to allow the accommodation of up to 15 balls without bursting. Eventually, the Ping-Pong balls may be replaced by hard plastic spheres, which give better performance as a result of less mutual friction. A laboratory glasswork drying frame is used to fill the balloons; the neck of a balloon is advanced some $5 \mathrm{~cm}$ over three pins of the frame (normally used to support an Erlenmeyer flask in upside-down position) that are held firmly together. When released, the backward springing pins keep the neck open, so that it becomes relatively easy to introduce balls in the balloon. Before starting, inflate the balloon a few times to increase its dead volume. Since a balloon cools on expansion, warm the empty balloon by holding it in your hand before inflating it again, to avoid condensation on the inner surface. A balloon pump is helpful. The balloon is removed from the springs and pumped, after each ball is inserted, so that the new ball can enter easily.

\section{Configurations}

Although well over 20 balls can be put in the balloon, the more interesting and easily recognizable coordination polyhedra occur with small numbers, for example (see figure), the familiar $n=4$ tetrahedron, the $n=5$ bipyramid, the $n=6$ octahedron (not shown), the rather unexpected $n=7$ pentagonal bipyramid, the $n=13$ cuboctahedron or its isomer, the $n=13$ icosahedron. All these structures form spontaneously — without premodelling - from a disordered cluster of balls that is forced in a small volume.

Let us watch the sequence of polyhedra more closely. The first isomers are encountered when $n=6$; both a regular octahedron and a tritetrahedron are frequently obtained. One may be converted to the other by pressing into the alternate shape. In the tritetrahedron only tetrahedral holes are found; by contrast, the octahedron encloses a single octahedral hole. Since the tritetrahedron may be considered an incomplete pentagonal bipyramid, its growth will eventually incorporate five-fold symmetry, which inhibits further growth into a periodic structure, but may be very important in the cluster domain. The octahedron, on the other hand, is part of the periodic $f c c$ structure (which can be seen by removing the eight corner atoms from an fcc unit-cell model). With more balls added, the octahedral hole becomes a rare feature, however, the structures being entirely composed of tetrahedra. With $n=7$, for example, the assembly almost invariably "crystallizes" in the very favorable pentagonal bipyramid; with $n=13$ (allowing a complete coordination shell) the icosahedral structure, consisting of 20 fused tetrahedra, is frequently obtained; again, five-fold symmetry is a dominant feature. The occurence of the $n=13 f c c$ structure, which may be identified by the square arrays on the surface, indicative of (half-) octahedral holes, is only rare. Moreover, the fcc structure is only marginally stable because the model readily transforms into the icosahedral arrangement.

\section{Experimental and Theoretical Studies that Illustrate the Model}

Many experiments and theoretical studies suggest the existence of pentagonal growth patterns in small aggregates. Thus, mass numbers of clusters of unusual stability and high frequency of occurrence (termed "magic" numbers) are strongly indicative of closed-shell arrangements with icosahedral or polyicosahedral symmetry $(2-4)$. The same conclusion is drawn from electron diffraction patterns (5). Icosahedral metal particles have been observed by electron microscopy $(6-10)$. Computer simulations have shown that closed-shell $f c c$ clusters of atoms, that interact through a Lennard-Jones potential, are unstable, and that a spontaneous transformation into the more favorable icosahedral arrangement is very likely (11-12). Similar results have been reported for molecular clusters (13).

The balloon-and-ball model can also be used to illustrate the structure of organometallic cluster-compounds, which are of great interest in the study of catalytic reactions. Here, small (transition-)metal atom clusters are surrounded by a coat of electron-donating ligands (phosphines, carbonyls, hydrides). In many cases the structures of the metal clusters (14), as well

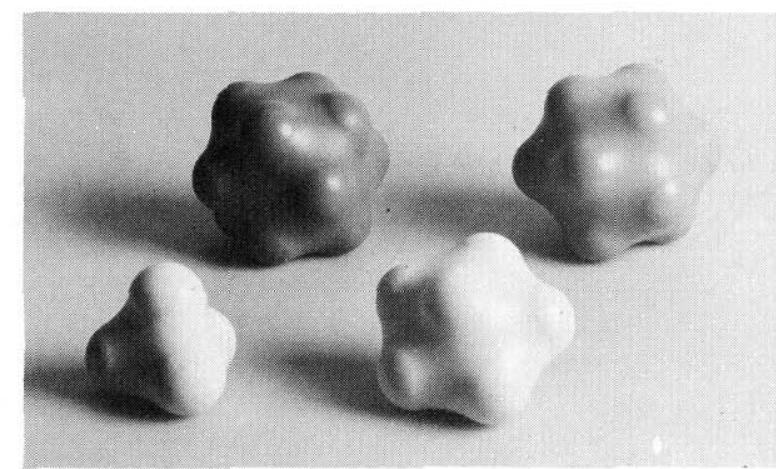

Some spontaneously "crystallizing" hard-sphere assemblies. From left to right: $n=4$ tetrahedron, $n=13 \mathrm{fcc}$ cuboctahedron, $n=7$ pentagonal bipyramid, and $n=13$ icosahedron. 
as that of the ligand coat (15), incorporate tetrahedral and icosahedral motifs, suggestive of small-scale close-packing without three-dimensional periodicity (although directional bonding may be important, as, for example, in the uncentered boron icosahedra (16)).

A biologically important example of aperiodic packing is found in the proteinic structure of small spherical viruses, where the coordination of small identical protein subunits, making up the virus coat or capsid, is the same as would result from the application of close-packing principles on a spherical surface: a regular polyhedron, based on the icosahedron (17).

\section{Literature Cited}

(1) Hoare, M. R., Adv. Chem. Phys., 40, 49 (1979)

(2) Kim, S. S., and Stein, G. D., J. Coll. Interf. Sci., 87, 180 (1982).

(3) Sattler, K., Mühlbach, J., Echt, O., Pfau, P., and Recknagel, E., Phys. Rev. Lett., 45, 821 (1980), 47, 160, 1121 (1981); Phys. Lett., 87A, 415 (1982).
(4) Powers, D. E. Hansen, S. G. Geusic, M. E., Puiu, A. C. Hopkins, J. B., Dietz, T. G. Duncan, M. A., Langridge-Smith, P. R. R., and Smalley, R. E., J. Phys. Chem., 86, 2556 (1982).

(5) Farges, J., de Feraudy, M. F., Raoult, B., and Torchet, G., J. Chem. Phys., 78, 5067 (1983).

(6) Allpress, J. G., and Sanders, J. V., Phyl. Mag., 10, 645 (1964); Surf. Sci., 7, 1 (1967)

(7) Mihama, K., and Yasuda, Y., J. Phys. Soc. Jap., 21, 1166 (1966).

(8) Ogawa, S., and Ino, S., J. Cryst. Growth, 13/14, 48 (1972).

(9) Hofmeister, B., Haefke, H., and Krohn, M., J. Cryst. Growth, 58, 507 (1982).

(10) Yacamàn, M. J., Romeu, D., Fuentes, S., and Dominguez, J. M., J. Chim. Phys., 78, 861 (1981).
Burton, J. J., J. Chem. Phys., 52, 345 (1970); Nature, 229, 335 (1971).

(11) Burton, J. J., J. Chem. Phys., 52, 345 (1970); Nature, 229, 335 (1971).
(12) Hoare, M. R., and Pal, P., Nature, 230, 5 (1971); 236, 35 (1972); J. Cryst. Growth, 17, 77 (1972); Adv. Phys., 20, 161 (1971); 24, 645 (1975); 32, 791 (1983).

(13) van de Waal, B. W., J. Chem. Phys., 79, 3948 (1983).

(14) Steggerda, J. J., Bour, J. J., and van der Velden, J. W. A., Recl. Trav. Chim. Pays-Bas, 101,164 (1982), and references cited therein.

(15) See, for example: Benfield, R. E., and Johnson, B. F. G., Transition Met. Chem., 6, 131 (1981), and references cited therein.

(16) See, for example: Sullenger, D. B., and Kennard, C. H. L.; Sci. Amer., 215 [1], 96 (1966).

(17) Klug, A., Nature, 303, 378 (1983). For an introductory account see: Kellenberger, E. Sci. Amer. 215 [12], 32 (1966). 